\title{
Ontology Management in an Event-Triggered Knowledge Network
}

\author{
Chen Zhou ${ }^{1}$, Xuelian Xiao ${ }^{2}$, Jeff DePree ${ }^{2}$, Howard Beck ${ }^{1}$, and Stanley $\mathrm{Su}^{2}$ \\ 1 Department of Agricultural and Biological Engineering, \\ University of Florida, Gainesville, Florida 32611 \\ 1-352-392-3797 \\ $\{$ czhou, hwb\}@ufl.edu \\ 2 Database Systems RD Center 458 CSE Building, \\ University of Florida, Gainesville, Florida 32611 \\ 1-352-392-2693 \\ $\{$ xxiao, jdepree, su\}@cise.ufl.edu
}

\begin{abstract}
This paper presents an ontology management system and ontology processing techniques used to support a distributed event-triggered knowledge network (ETKnet), which has been developed for deployment in a national network for rapid detection and reporting of crop disease and pest outbreaks. The ontology management system, called Lyra, is improved to address issues of terminology mapping, rule discovery, and large ABox inference. A domain ontology that covers the concepts related to events, rules, roles and collaborating organizations for this application in ETKnet was developed. Terms used by different organizations can be located in the ontology by terminology searching. Services that implement knowledge rules and rule structures can be discovered through semantic matching using the concepts defined in the ontology. A tableau algorithm was extended to lazy-load only the needed instances and their relationships into main memory. With this extension, Lyra is capable of processing a large ontology database stored in secondary storage even when the ABox cannot be entirely loaded into memory.
\end{abstract}

\section{Introduction}

Government organizations worldwide are facing complex problems such as illegal immigration, terrorism, disease outbreaks, and natural disasters. Effective resource sharing, collaboration and coordination among government organizations are needed to solve these and other complex problems. They need to share not only data and application system functions, but also human and organizational knowledge useful for decision support, problem solving and activity coordination. The technology of sharing distributed, heterogeneous data has been extensively studied, but an effective way of sharing knowledge among collaborating organizations is still lacking.

The distributed event-triggered knowledge network (ETKnet) is an event and rule-based system, which was developed for deployment in a national network

L. Aroyo et al. (Eds.): ESWC 2010, Part I, LNCS 6088, pp. 410 424, 2010.
(C) Springer-Verlag Berlin Heidelberg 2010 
for rapid detection and reporting of plant disease and pest outbreaks. The National Plant Diagnostic Network (NPDN1) has been organized to strengthen the homeland security protection of the United State's agriculture by facilitating quick and accurate detection of disease and pest outbreaks in crops. NPDN has developed a general standard operating procedure (SOP [1] ) to respond to a pest or disease outbreak. This SOP details the steps to be taken when such a biosecurity event takes place. ETKnet uses events and different types of knowledge rules and rule structures to implement the SOP.

ETKnet is used to capture, manage, and apply the multifaceted knowledge embedded in organizational as well as inter-organizational policies, regulations, constraints, processes and operating procedures. A common means of representing knowledge is to use knowledge rules [2. Three types of knowledge rules have been found to be useful in many applications [34: integrity constraints [5], logic-based derivation rules [6], and condition-action rules [78. One possible approach for the interoperability of these heterogeneous rules is to choose one rule type as a common format and convert the other rule types into this chosen representation 9. This approach sounds attractive because it only needs a single rule engine to process all the converted rules. However, since different types of rules have significant semantic disparities, converting a rule from one representation to another may lead to loss of information. Another possible approach is to build wrappers around different types of rule engines [10, and provide a common interface to enable these rule engines to exchange the data generated by rules. This approach is not ideal either, because it will result in a very complex system that is difficult to operate and maintain. In our work, we extend the condition-action rule to allow the specification of a structure of operations that models a sharable process in the action clause to allow the process to be conditionally enacted. These operation structures can have the structural constructs selected from workflow process definition languages such as WPDL 11 and BPEL[12]. A single knowledge and process specification language is used for collaborating organizations to define different types of rules and rule structures that incorporate processes.

When organizations collaborate, it is usually to solve specific problems. Due to security and practical reasons, opening the database up in its entirety for access by other organizations is not a safe option. Thus it is important to devise a framework that allows organizations to share only those data and knowledge pertaining to specific problems. Our approach for achieving data and knowledge sharing is to augment an event subscription and notification system with knowledge sharing and processing capabilities. An event is anything of significance to collaborating organizations (e.g. an arrest, a terrorist incident, the detection of a disease, a special state of a database, a signal from a sensor, etc.) that occurs at a particular point in time. Data associated with an event occurrence (i.e., the event data) may trigger the processing of distributed rules and rule structures, which can produce new data or modify the event data. The new event data may trigger other rules and rule structures. Thus, multiple rounds of event data

${ }^{1}$ NPDN: National Plant Diagnostic Network, http://www.npdn.org 
transmission, rule processing, and data aggregation may take place in order to produce all the data that are pertinent to the event occurrence.

There are a number of knowledge sharing challenges in such a distributed heterogeneous environment. The first issue is the heterogeneous terminology problem. Collaborating organizations may use their own terms to specify their events, event data, and rules, as well as the roles that their users play. The meanings of these terms need to be properly defined. Technologies for effective searching and processing of distributed, heterogeneous events, rules, roles and organizations are also needed. Our approach for meeting these needs is to develop a domain-specific ontology, which covers the concepts that are related to events, rules, roles, and organizations in the ETKnet system. Terms are mapped to ontology concepts that define the meaning of the terms to address the terminology discrepancy. The Lyra ontology management system is used to maintain the domain specific ontology and the terminology mapping. The second issue is the rule service's discovery. Collaborating organizations often want to find the applicable rules for certain event data. A rule or rule structure is applicable for an event if the input data it's expecting is a subset of the event data. Knowledge sharing is facilitated through a publish and subscription model. An ontology-enhanced registry is developed for the discovery of events, rules, roles and organizations. The third issue is the scalability problem. The ETKnet system may involve huge amounts of event data. Lyra needs to support large secondary storage management and a scalable inference engine. To meet this requirement, we have extended the tableau algorithm to lazy-load only the needed instances into main memory. Thus, a large ABox can be utilized even it cannot be fully loaded into memory.

In the following sections, we briefly describe the implemented ETKnet system and use a sample scenario taken from the implementation of SOP to explain ETKnet's distributed event and rule processing process so that the reader will know how the ontology support comes into play. We then present the domainspecific ontology defined for the implementation of the SOP, and present the ontology management system and its lazy-loading algorithm. Related work and a conclusion are given in the last two sections.

\section{Background}

\section{$2.1 \quad$ ETKnet}

ETKnet has a peer-to-peer server architecture as shown in Fig. 1] All collaborating organizations have identical subsystems installed at their sites. Each site creates and manages its own events, rules, rules structures and operations. Their specifications are registered at the host site of a collaborative federation. The host maintains a repository of these specifications. A user interface is used at each site to define sharable events, rules, rule structures, application system operations and operation structures. 


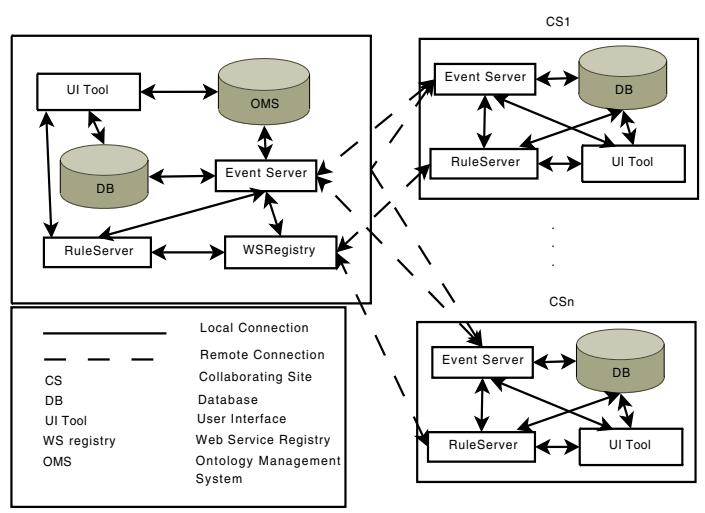

Fig. 1. System Architecture

Through a relatively simple progress, the defined rules and rule structures are translated into code and wrapped as web services for distributed knowledge and process sharing and interoperation. This approach avoids the use of multiple rule engines and workflow/process management systems, as well as the problem of rule-to-rule conversions.

The system supports three types of rules: logic-based derivation rule adopted from RuleML [13, constraint rule patterned after [14, and action-oriented rule 8]. A rule structure is a directed graph with different types of rules as nodes. These nodes are connected by link split, and-join, and or-join constructs, which specify sequential and parallel processing of rules and two alternative ways of rule synchronization. When a shared rule or a rule structure is defined, a relatively simple progress is used by the Rule Server at that site to convert it into program code, wraps the code as a Web service, stores the rule information in the local database and registers the generated Web service with the WSRegistry at the host site. This approach avoids the use of multiple rule engines and workflow/process management systems, as well as the problem of rule-to-rule conversions.

The Event Server is responsible for storing information about events defined at that particular site and information about event subscribers. Events defined at all sites are registered with the host site. Users of all organizations can browse or query the registry to subscribe to the event. They can also define triggers to link distributed events to distributed rules and rule structures. In both cases, they are "explicit subscribers". Triggers can also be generated automatically and dynamically by the system for those sites that contain "applicable" rules and rule structures. A rule or rule structure is applicable to an event if its input data requirement is a subset of the event data. The organization that defines the applicable rule or rule structure is an "implicit subscriber" to the event. The occurrence of an event will cause event data to be sent to explicit and implicit subscribers and be process by their rules and rule structures. 


\subsection{Sample Scenario in NPDN}

The collaborative federation that serves as the application domain is NPDN. NPDN links plant diagnostic facilities across the United States and several US territories. The following is a sample submission scenario in the NDPN system (Figure 2). When a suspect sample of plant tissue suspected of having a new disease or insect is submitted to the NPDN disease diagnostics triage lab, an event, Presumptive-Positive-Sample-Observed occurs (Step 1, labeled E1 in the figure) E1 at the NPDN Triage Lab causes the event data containing the sample information to be sent to APHIS Lab and NPDN Regional Hub Lab (Step 2). This event data is sent to both sites as an XML document. These labs have applicable rules that can make use of the event data to provide some more relevant information. APHIS performs a preliminary diagnosis on the sample (Step 3). The NPDN Regional Hub Lab informs the appropriate personnel of the sample status (also Step 3). These procedures at the NPDN Triage Lab and the NPDN Regional Hub Lab are modeled using heterogeneous rules and rule structures. Details of these procedures are given later in this section.

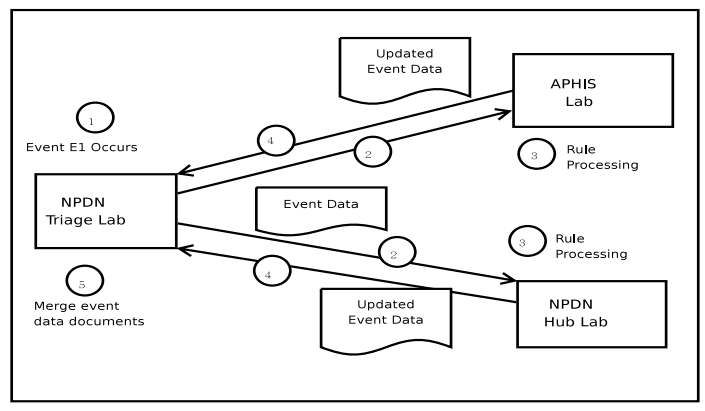

Fig. 2. Distributed Event and Rule Processing

The invocation of the applicable rules and rule structures in both the APHIS CDD Lab and the NPDN Regional Hub Lab may produce new data or modify the existing data. The new data and updates are then sent back to the NPDN Triage Lab as modifications to the original event data document (step 4) and are merged with the original event data (step 5). The new version of event data is sent (not shown in the figure) to the APHIS Lab and NPDN Regional Hub Lab to begin a second round of rule processing if there are applicable rules. Thus, multiple rounds of event data transmission, rule processing and data aggregation can take place as event data is dynamically changed by rules. The algorithms for aggregating event data to produce a new version of event data, and for avoiding cyclic processing of distributed rules can be found in [15].

Figure 3 describes the rule structure executed at the NPDN Triage Lab upon receiving a sample. The rule structure has both link and join relationships. The first rule, NTLR1, is concerned with acknowledging the receipt of the sample 


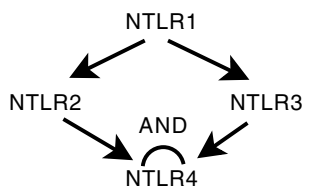

Fig. 3. Rule Structure upon Sample Received Event

by the sample submitting entity. It assigns a unique id to the sample, and asks the lab diagnostician to perform a preliminary diagnosis on the sample. Rule NTLR2 checks whether the sample has been reviewed by a diagnostician. If this is the case, it is classified as 'presumptive positive'. Meanwhile, NTLR3 instructs and guides the lab staff on how to contact the APHIS-CDD and NPDN hub, and how to store the sample securely. Then, an NPDN Sample event is posted in the NTLR4 to inform the system that a portion of the sample has been sent to the APHIS-CDD lab and NPDN Hub lab. For space reasons, we do not include the rules or rule structures at other NPDN labs.

\section{Ontology for ETKnet System}

\subsection{Rules Definition and Matching}

To support data interoperability and registry discovery, a domain ontology was defined to cover the concepts related to events, rules, roles and organizations in ETKnet. The ETKnet ontology defined in the NPDN domain contains the metadata definition for the rules, events, roles, and organizations in the context of the SOP. Each rule individual is an instance of CAARule, IntegrityRule, DerivativeRule, or their subclasses. In our system, each of these rules are converted to Java code, and wrapped as web services. The rule web service in the registry concerns only the input and outputs, and internally rules process actions. Hence the properties input, output, and action are defined in the rule ontology. For example, table 11 shows each rule's definition in Fig. 3. The NTLR1 rule becomes an individual in the ontology and is an instance of CAARule. Its input property has value StateOfOrigin, and its output property has values of SampleID and SampleExamined. Here are all NTLR rules definition according to the table 1 .

NTLR $1 \in C$ AARule $\sqcap$ Binput.StateOfOrigin $\sqcap \exists$ output.(SampleID $\sqcup$ SampleExamined) NTLR2 $\in$ DerivationRule $\sqcap \exists$ input.SampleExamined $\sqcap \exists$ output.Classification NTLR3 $\in$ CAARule $\sqcap$ ヨinput.SampleExamed NTLR4 $\in$ CAARule $\sqcap \exists$ output.(Sample $\sqcup$ SampleId $\sqcup$ State)

These rule definitions are stored in the Lyra OMS, and the ontology reasoner can utilize these metadata definitions for rule discovery. For a given inquiry $\mathrm{P}$, the reasoner returns the set of all the rule individuals which are compatible with $\mathrm{P}$. The inquiry $\mathrm{P}$ and the rule individual $\mathrm{r}$ are compatible iff $\mathrm{r}$ is an instance of 
Table 1. NPDN Triage Rules on Sample Received

\begin{tabular}{|l|l|l|l|l|}
\hline Name & Type & Input & Output & Action \\
\hline NTLR1 & CAARule & StateOfOrigin & $\begin{array}{l}\text { SampleID, } \\
\text { SampleExamined }\end{array}$ & $\begin{array}{l}\text { 1. Acknowledge Receipt } \\
\text { 2. Assign sample ID } \\
\text { and examine sample }\end{array}$ \\
\hline NTLR2 & DerivationRule & SampleExamined & Classification & \\
\hline NTLR3 & CAARule & SampleExamined & & Store sample securely \\
\hline NTLR4 & CAARule & & $\begin{array}{l}\text { Sample, } \\
\text { SampleID, } \\
\text { StateOfOrigin }\end{array}$ & $\begin{array}{l}\text { 1. Post NPDN_Sample_Event } \\
\text { 2. Send sample to APHIS-CDD } \\
\text { 3. Send sample to NPDN Hub }\end{array}$ \\
\hline
\end{tabular}

$\mathrm{P}$, and r's input and output are compatible with P's input and output. A rule or rule structure is applicable for an event if its input data requirement is a subset of the event data. Algorithm 1 shows the algorithm for the rule web service's discovery. The user enters the rule's concept P, input concept I, and output concept $\mathrm{O}$ for the algorithm. Since all the result candidates must be an instance of $\mathrm{P}$, the algorithm realizes the rule concept $\mathrm{P}$ and retrieves its instances as candidates. Among this candidate set, a comparison is made between each instance's input/output with the user's required input/output. The matching score is calculated after these comparisons are completed. An EXACT match implies that the required input $R_{I}$ /output $R_{O}$ and the candidate's input $S_{I}$ /output $S_{O}$ are equivalent concepts. It has the highest score, since this indicates that the inputs and the requirement are exactly the same. A SUBSUME matching denotes that the required input $R_{I}$ is a subconcept of the candidate's input $S_{I}$, or that the candidate's output $S_{O}$ is a subconcept of the required output $R_{O}$. It has a lower score, since the rule service requires fewer inputs than have been specified in the request. This guarantees that the rule will be executable with the provided inputs. In addition, the rule is expected to return more specific output data whose semantics are exactly the same or very close to what has been requested by the user. Other matches are treated as failures. According to this algorithm, the user's query is converted to the description logic (DL) concept realization problems, and answered by Lyra's inference engine. This algorithm is currently used for purpose of rule discovery. The result set does not have any specific order for rules with the same score. All the matched services are proposed for checking as candidate services.

Here is an example of the rule web service's reasoning. The user wants to find the CAA rule whose input includes the State class, and whose output contains SampleID and SampleExamined. The inquiry concept P can be expressed as:

$$
P=C A A R u l e \sqcap \exists \text { input.State } \sqcap \exists \text { output.(SampleID } \sqcup \text { SampleExamined) }
$$

According to the example scenario, the candidate set by realization contains NTLR1. Meanwhile its input and output are compatible with the user's requirement, hence it is returned in the result set. 


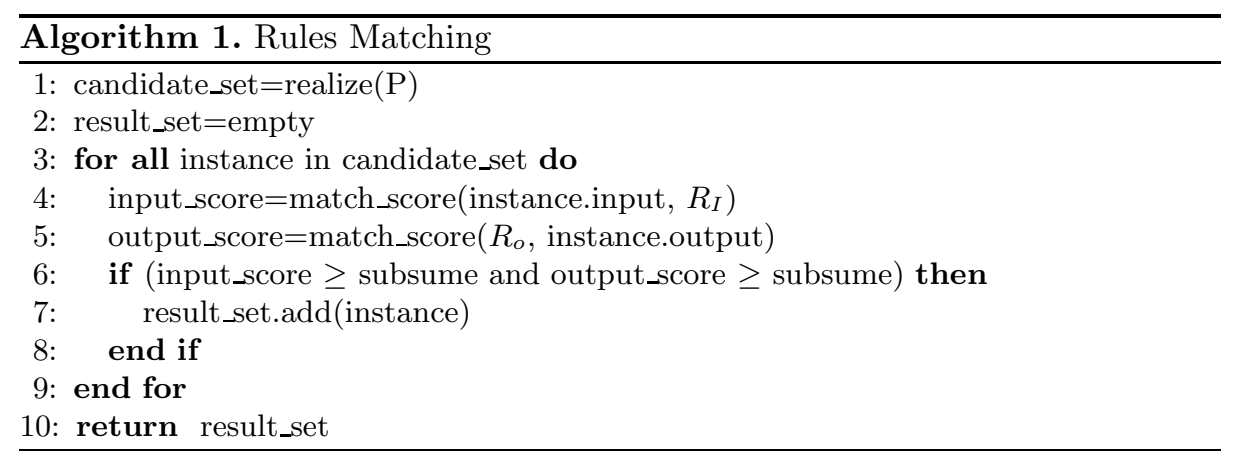

\section{Lyra Ontology Management System and Lazy-Loading Algorithm}

An ontology management system (OMS) is a database framework for creating, storing, modifying, and querying ontology information. Lyra, designed as an OMS, is used in a number of different application projects, such as the ETKnet project, digital library projects, and agricultural decision support systems [16]. In this section we will use the ETKnet NPDN project as an example and provide more detailed information about Lyra.

\subsection{Terminology Mapping}

Terminology mapping is integrated with Lyra's objects. Figure 4 shows the main data definition language for Lyra. The top portion of the figure creates a mapping from terminology to the ontology objects. It has a similar data definition as Wordnet [17. Among them, a term is a single word or short phrase, which can have one or more specific meanings. Each of the specific meanings is represented by a word sense. Every SynSet represents a single concept and contains a set of terms that have the same meaning (synonyms). Different terms can point through word senses to the same SynSet.

SynSet's formal meaning is represented directly using an ontology object. That is, Each SynSet is mapped to exactly one object in the ontology. Lyra provides the lookup API to find the SynSet's ontology definition, and vice verse. This allows the reasoning engine to perform inferences according to the ontology's formal definition. For example, the relationships between SynSets can be checked by the reasoning engine. Two most typical relationships in SynSets are hyponym (subclass) and hypernym (superclass).

Lyra provides an API to search through the terminologies and get the SynSets. When an organization needs to define or search for some event data, Lyra's terminology information can be searched to get the candidate SynSets, or the hierarchy of the event data ontology can be browsed to identity an event.

For example, the NPDN ontology contains definitions of events, roles, and organizations specified in the SOP. In a collaborative environment, terms used 


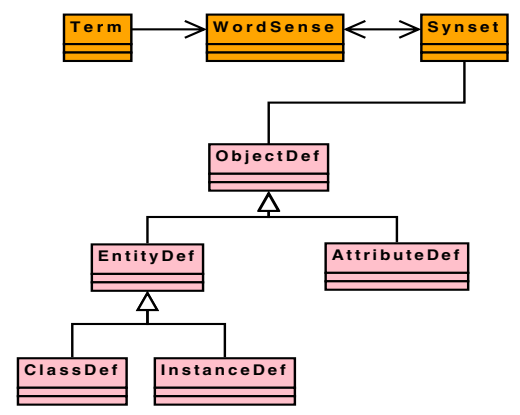

Fig. 4. Overview of Lyra Data Definition Language

by different organizations to describe the same thing can be quite different. To resolve this terminology level discrepancy, Lyra is used to maintain a mapping between the organizations' terminology and the ontology. Based on the mapping, Lyra can find the terminology's matched concepts for the user. The user does not need to know the exact concepts in the system, only terminology previously defined by his or other organizations. In the event that a concept needed by the user does not exist in the ontology, it must be added, along with associated terminology, under the supervision of an ontology administrator.

\subsection{Lyra Data Definition Language and Reasoning Engine}

Lyra's data definition language is designed according to description logic. It implements DL $\mathcal{S H \mathcal { Q }}$ and defines its own syntax for it. Table 2 shows the main language constructors in Lyra, as well as their interpretations. An interpretation $I=\left(\triangle^{I},{ }^{I}\right)$ consists of a mapping from every concept to a set $\triangle^{I}$ and from every role to a subset of $\triangle^{I} \times \triangle^{I}$ such that, for all concepts $C, D$, roles $R, S$, and non-negative integers $n$, the equations in table 2 are satisfied, where $\# M$ denotes the cardinality of a set $M$. Since OWL-DL's semantic model is based on description logic 18, most of the constructors in OWL-DL have a mapping to the constructors in Lyra. To facilitate knowledge sharing between different research groups, we have developed an OWL conversion tool to support the interoperability of OWL and Lyra.

The main objects in Lyra's data definition language are ClassDef, AttributeDef, and IndividualDef. They are presented at the bottom of Figure 4. They correspond to the concept, role, and individual definitions in the description logic respectively. Each of these objects contains an object identity and a display term. These are defined in ObjectDef, a parent class of ClassDef, AttributeDef, and IndividualDef, as shown in Figure 4. Object identity (OID) is used to uniquely identify the object in the Lyra object database. The display term represents the gloss of the object. All the objects that inherit ObjectDef in turn have these properties.

Lyra's object database supports two types of retrieval functions: SynSet retrieval and object retrieval. SynSet retrieval uses the term as the input, and 
Table 2. Interpretation of Lyra Definition Language

\begin{tabular}{|l|l|}
\hline Lyra & Interpret \\
\hline Inverse & $\left(R^{-}\right)^{I}=<x, y>\mid<y, x>\in R^{I}$ \\
\hline Conjunction & $(C \sqcap D)^{I}=C^{I} \cap D^{I}$ \\
\hline Disjunction & $(C \sqcup D)^{I}=C^{I} \cup D^{I}$ \\
\hline Exists restriction & $(\exists R . C)^{I}=\left\{x \mid \exists y .<x, y>\in R^{I}\right.$ and $\left.y \in C^{I}\right\}$ \\
\hline Value restriction & $(\forall R . C)^{I}=\left\{x \mid \forall y .<x, y>\in R^{I}\right.$ and $\left.y \in C^{I}\right\}$ \\
\hline$\geq \mathrm{n}$ number restriction & $(\geq n R . C)^{I}=\left\{x \mid \#\left\{y .<x, y>\in R^{I}\right.\right.$ and $\left.\left.y \in C^{I}\right\} \geq n\right\}$ \\
\hline$\leq \mathrm{n}$ number restriction & $(\leq n R . C)^{I}=\left\{x \mid \#\left\{y .<x, y>\in R^{I}\right.\right.$ and $\left.\left.y \in C^{I}\right\} \leq n\right\}$ \\
\hline
\end{tabular}

returns all related SynSets for this term. Users can identify whether the SynSet is the desired one by checking its definition in the ObjectDef. Object searching uses the OID as the input, and returns the ObjectDef definition for the user.

Lyra's reasoning engine provides consistency checking and realization services based on description logic semantics. The TBox contains the ontology's definition information. The ABox is a list of assertions about individuals and their memberships. The core of the reasoning engine is a tableau reasoner for description logic that can check the consistency of a concept. Many of our projects require the datatype inference in the realization tasks, hence we support number, string and many other XML schema datatype value inferences.

Integration has been carried out between the Lyra object database and the reasoning engine. The object database is treated as a persistent layer for storing and retrieving object definitions. The reasoner needs to retrieve the ClassDef, AttributeDef, and IndividualDef definitions from Lyra's persistent layer first, and then convert them into normalized format for the reasoner's processing. The concept's unique string object id (SOID), or the automatically assigned unique integer id (OID) can be used as the concept's unique representation in the DL reasoner. SOID representation is more human friendly for the result interpretation, while the OID representation provides better performance for inference.

\subsection{Lazy-Loading Tableau Algorithm}

When an ontology has a normal sized TBox and a large ABox, the ABox can be a bottleneck for the memory based tableau algorithm. We invent the lazy-loading algorithm to address this problem. The main idea in this algorithm is to avoid bringing individuals into main memory unless and until needed by the tableau algorithm. The $\forall, \leq$, or $\forall+$ rules are modified to lazy-load the individuals at runtime. In the lazy-loading algorithm, the idea is to identify when and where it is necessary to load the individuals' type definition and neighboring relationship information in the tableau algorithm's rules. Let's take the tableau algorithm for DL $\mathcal{S H \mathcal { I }}$ as the example. Suppose that $a$ and $b$ are named individuals in $A, x$ is an unnamed individual, $C$ is a concept in $A$, and $R$ is a role. A new relationship assertion $R(a, b)$ may influence the result of the tableau algorithm only if one of the following two conditions holds: 
- The relationship assertion is used to trigger new applications of tableau rules that alter the ABox. For example, a role assertion can be used to add new membership assertions about the individuals by the application of the $\forall$ rule on a if $a$ has the concept label $(\forall R . C)$, and $b$ is an R-neighbor of $a$.

- The relationship assertion is involved in clash detection due to the violation of a maximum cardinality restriction. Here is an example: if $a$ has label $\leq n R$ and $b$ is one of $n+1$ mutually distinct R-neighbors of $a$, then $R(a, b)$ is needed to be loaded, and can cause the clash in tableau.

These conditions can only hold by either the application of the $\forall, \leq$, or $\forall+$ or the presence of a maximum cardinality constraint. In contrast, the $\exists$ and $\geq$ do not need to know the real role assertions $R(a, b)$, since they artificially create new role assertions and new unnamed individuals for satisfying the $\exists R$. $C$ and $\geq n R$ constraints. From these observation, we know that the role assertion $R(a, b)$ and the range instance can be delayed until the tableau algorithm requires such information. This helps to load only necessary information into memory.

Table 3. The Lazy Loading Tableau Rules

\begin{tabular}{|c|c|}
\hline$\forall$-rule & $\begin{array}{l}\text { if } \mathrm{x} \text { is not loaded, lazy load } \mathrm{x} \text { from secondary storage } \\
\text { if } 1 . \forall S . C \in \mathcal{L}_{i}(x) \text {, x is not indirectly blocked, and } \\
\text { 2. there is an S-neighbour y of x with } C \notin \mathcal{L}_{i}(y) \\
\text { then } \mathcal{L}_{i}(y) \rightarrow \mathcal{L}_{i}(y) \cup\{C\}\end{array}$ \\
\hline$\forall_{+}$-rule: & $\begin{array}{l}\text { if } \mathrm{x} \text { is not loaded, lazy load } \mathrm{x} \text { from secondary storage } \\
\text { if } 1 . \forall S . C \in \mathcal{L}_{i}(x) \text {, x is not indirectly blocked, and } \\
\text { 2. there is some } \mathrm{R} \text { with } \operatorname{Trans}(R) \text { and } R \text { 函 } S, \\
\text { 3. there is an R-neighbour y of x with } \forall R . C \notin \mathcal{L}_{i}(y) \\
\text { then } \mathcal{L}_{i}(y) \rightarrow \mathcal{L}_{i}(y) \cup\{\forall R . C\}\end{array}$ \\
\hline S-rule: & $\begin{array}{l}\text { if } \mathrm{x} \text { is not loaded, lazy load } \mathrm{x} \text { from secondary storage } \\
\text { if } 1 . \leq n S . C \in \mathcal{L}_{i}(x), \mathrm{x} \text { is not indirectly blocked, and } \\
\text { 2. } \sharp S_{i}^{\mathcal{F}}(x, C)>n \text {, there are } \mathrm{S} \text {-neighbours } \mathrm{y}, \mathrm{z} \text { of } \mathrm{x} \text { with not } y \neq z, \\
\mathrm{y} \text { is neither a root node nor an ancestor of } \mathrm{z} \text {, and } C \in \mathcal{L}_{i}(y) \cap \mathcal{L}_{i}(z) \\
\text { then } 1 . \mathcal{L}_{i}(z) \rightarrow \mathcal{L}_{i}(z) \cup \mathcal{L}_{i}(y) \text { and } \\
\text { 2. if } \mathrm{z} \text { is an ancestor of } \mathrm{x} \\
\quad \text { then } \mathcal{L}_{i}(\langle z, x\rangle) \rightarrow \mathcal{L}_{i}(\langle z, x\rangle) \cup \operatorname{lnv}\left(\mathcal{L}_{i}(\langle x, y\rangle)\right) \\
\text { else } \mathcal{L}_{i}(\langle x, z\rangle) \rightarrow \mathcal{L}_{i}(\langle x, z\rangle) \cup \mathcal{L}_{i}(\langle x, y\rangle) \\
\text { 3. } \mathcal{L}_{i}(\langle x, y\rangle) \rightarrow \emptyset \\
\text { 4. Set } u \neq z \text { for all } \mathrm{u} \text { with } u \neq y\end{array}$ \\
\hline$\leq$ & $\begin{array}{l}\text { if } \mathrm{x} \text { is not loaded, lazy load } \mathrm{x} \text { from secondary storage } \\
\text { if } 1 . \leq n S . C \in \mathcal{L}_{i}(x) \text {, and } \\
\text { 2. } \sharp S_{i}^{\mathcal{F}}(x, C)>n \text { and there are two S-neighbours y, z of x } \\
\quad \text { which are both root nodes, } C \in \mathcal{L}_{i}(y) \cap \mathcal{L}_{i}(z) \text {, and not } y \neq z \\
\text { then Merge }(\mathrm{y}, \mathrm{z})\end{array}$ \\
\hline
\end{tabular}

Applying the lazy-loading algorithm to the Lyra reasoner, we assume that the ontology in the Lyra OMS is consistent, and we have loaded the classified TBox during preprocessing. When a query is issued to Lyra, the query concept is 
classified so that its equivalent concepts' and sub-concepts' instances are added into the result set. The query's direct super concepts' instances are added into the unknown set for further testing by the tableau algorithm. After adding the obvious individuals from the unknown set into the result set, the realization process starts on the remaining unknown set linearly or bulkily. Here we control the bulk size of this subset to fit in the memory. During the completion strategy in tableau algorithm, we modified the $\forall, \leq$, and $\forall+$ rules to lazy-load the individuals from Lyra secondary storage in the runtime only when necessary. This is shown in the Table 3. When any of these rules are triggered, it checks whether the named individual has already been loaded into memory. If not, the lazy-load process will load it in and process the initialization. After that, the rule continues and tableau algorithm will stop when either all the branches have conflicts or a satisfied branch is found.

\subsection{Proof of Lazy-Loading Tableau Algorithm}

In the following we show that the lazy-loading tableau algorithm for DL $\mathcal{S H \mathcal { C }}$ generates the correct results. A lazy-loading ABox is an ABox $A^{\prime}$ corresponds to the original SHIQ ABox $A$, but the nodes in $A^{\prime}$ (except the root nodes) are loaded only at runtime when $\forall, \leq$, and $\forall+$ rules are triggered in the tableau algorithm. Here we want to prove: to the lazy-loading ABox $A^{\prime}$ obtained by applying the lazy-loading to $A, A$ is consistent w.r.t. $T$ and $R$, iff $A^{\prime}$ is consistent. This proof is straightforward. For any inference branches in the tableau algorithm, the processed subset of ABox $A^{\prime}$ is simply retrieved from the original ABox $A$. If there is any conflicts in $A$, it will also be found in $A^{\prime}$. Hence if $A^{\prime}$ is consistent, the original $A$ is also consistent. If there is no conflicts in $A$, they will not be found in $A^{\prime}$ either. Hence if $A^{\prime}$ is not consistent, the original $A$ is also not consistent.

According to this proof, we can apply the tableau algorithm directly on the lazy ABox A' instead of original ABox A for the inference. The classification and realization are based on the tableau algorithm, so that we can apply them on $A^{\prime}$ directly as well. Our realization process requires that the whole TBox has been preprocessed and loaded in the memory. After the preprocessing, lazy-loading ABox $A^{\prime}$ has only the candidate individuals loaded while leaving all other individual information in the Lyra secondary storage. The tableau algorithm starts from this small candidate set in the A'. When $\forall, \leq$, and $\forall+$ rules are triggered in the tableau algorithm, the individual's definition and neighboring relationship will be lazy-loaded into $A^{\prime}$. In the worst case, the tableau algorithm may load the whole ABox A into the memory, but normally only a small portion of the ABox $A$ is needed during this process. Hence we can perform the inferencing on a lot of ABoxes that cannot fit into memory by the traditional tableau algorithm. In some cases (actually many cases in this application), there is only datatype reasoning involved in the query, and so the lazy-loading ABox needs only to load the individual's own data information.

A number of optimizations are adopted in the lazy-loading algorithm to improve the performance. Each individual in the unkown candidate set is prechecked to find obvious conflicts to avoid invoking the tableau algorithm. The 
cached lazy-loading ABox is kept in different invocations of the tableau algorithm to get improved in-memory performance. When the memory usage reaches a threshold, the lazy-loading ABox is reinitialized to free unneeded memory usage. Our experiments on the $\operatorname{LUBM}(5,0)$ [19] database, as well as other applications we have tested, confirm that lazy-loading produces the correct result. The lazy-loading tableau algorithm has runtime IO and instance insertion's overhead. The overhead depends on the query type and the loaded instance number. The time for various queries in the LUBM differs from one second to tens of seconds. The time for query in our publication database is within a few seconds, which is acceptable for normal end users.

\section{Related Works}

ETKnet system is a distributed rule system to support processing and sharing of knowledge. Web services are adopted in our system to improve the interoperability in the heterogeneous environment. In the last few years, a number of works have been reported for semantic web services. For example, OWL-S [20] presents specific ontology designed for web services and their process. Matchmaking algorithms, such as 21], help to discover the proper services according to their input, output, precondition, and effect (IOPE). Web Servcies Modeling Ontology (WSMO) [22] has some similarity with OWL-S, and provides additional mediator concept for service integration. Our system focuses more on the rules and their interoperability in the distributed environment. There is no specific global process defined in ETKnet. Event data will trigger the rules to process and share the knowledge.

Much research has been done on logic programming. SWSL 23] is a language for describing the concept of web services. The logic programing part of it, SWSL-Rules, is used to describe the services specification. SILK [24] is a recent work to study the proper enhancement on existing rule specification to achieve scalable and expressive semantic rules. These works provide the building blocks for annotation and inference of service descriptions. In contrast, our system studies how rules can be inferenced in a distrbuted way, and how the domain knowledge is used to sovle the interoperability problems.

The ontology management system plays a key role for data processing in a heterogeneous environment. One important issue in our system is scalable ABox reasoning. A number of previous works have dealt with this problem. Instance store 25] presents a role free ABox for instance retrieval. Its ABox allows only type assertions but not property assertions, while Lyra supports the normal DL constructors. DL-Lite [26] supports a subset of OWL-DL called DL-Lite. All the queries in DL-Lite can be translated into a set of SQL queries for processing. We supports the full OWL-DL, which is useful in some projects. SHER [27] stores instance data in the database, and creates a small summary ABox from the original ABox for query processing. It supports OWL-DL minus datatypes and nominals. This engine's ability is quite similar to the Lyra system. However, datatype inference is supported in our system to meet the projects' requirement. 
By lazy loading the ABox instance during the tableaux procedure, Lyra helps to deal with large ABox that cannot be fit in the main memory.

\section{Conclusion}

This paper describes the approaches taken in ETKnet to achieve the sharing of distributed events and rules defined by collaborating organizations. Three types of commonly used rules and rule structures with imbedded application operations and operations structures are used to specify organizational and interorganizational policies, constraints, regulations, processes and procedures. The distributed event and rule processing technique of ETKnet enables the activation of all the applicable rules and rule structures defined by collaborating organizations and ensures that these organizations receive all the data that are relevant to an event occurrence. The Lyra ontology management system manages the domain-specific ontology and enables terminology searching for events, roles and organizations and semantic searching for sharable rules. Rule web services' discovery assists the organizations to find shared rules in the ETKnet system. The lazy-loading algorithm helps to manage a large ABox even when it cannot fit main memory.

\section{Acknowledgements}

This project is supported by NSF under grant number IIS-0534065.

\section{References}

1. NPDN Scenario SubCommittee: The National Plant Diagnostic Network Standard Operating Procedure for APHIS-PPQ Pest of Concern Scenario - General SOP (2005)

2. Sowa, J.: Knowledge Representation: Logical, Philosophical and Computational Foundations. Brooks Cole Publishing Co., Pacific Grove (2000)

3. Group, B.R.: Defining Business Rules - What Are They Really? http://www.businessrulesgroup.org/first_paper/br01c0.htm

4. Grosof, B., Rouvellou, I., Degenaro, L., Chan, H.Y., Rasmus, K., Ehnebuske, D., McKee, B.: Combining different business rules technologies: a rationalization. In: OOPSLA Workshop on Best-practices in Business Rule Design and Implementation (2000)

5. Ullman, J.: Principles of Database Systems, 2nd edn. Computer Science Press, Rockville (1982)

6. Ullman, J.: Principles of Database and Knowledge-Base Systems. Computer Science Press, Rockville (1988)

7. Bry, F., Eckert, M., Ptrnjan, P., Romanenko, I.: Realizing business processes with ECA rules: benefits, challenges, limits. In: Int. Workshop on Principles and Practice of Semantic Web Reasoning, pp. 48-62 (2006)

8. Widom, J., Ceri, S.: Active Database Systems, Triggers and Rules for Advanced Database Processing. Morgan Kaufmann, San Francisco (1996) 
9. Bassiliades, N., Vlahavas, I., Elmagarmid, A.K.: E-device: an extensible active knowledge base system with multiple rule type support. IEEE Transactions on Knowledge and Data Engineering 12, 824-844 (2000)

10. Rosenberg, F., Dustdar, S.: Towards a distributed service-oriented business rules system. In: IEEE Third European Conference on Web Services (November 2005)

11. Workow Management Coalition: Workow management coalition workow standard: Interface 1 process denition interchange process model (wfmc-tc-1016). Technical report, Lighthouse Point, Florida (1999)

12. IBM, BEA Systems, Microsoft, SAP AG, and Siebel Systems: Business Process Execution Language for Web Services version (BPEL4WS) 1.1. (2003), http://www-106.ibm.com/developerworks/library/ws-bpel/

13. RuleML committee: The rule markup initiative, http://www.ruleml.org

14. Object Management Group: Object constraint language specification (2001), http://www.omg.org

15. Degwekar, S., DePree, J., Beck, H., Thomas, C., Su, S.: Event-triggered data and knowledge sharing among collaborating government organizations. In: International Conference on Digital Government Research, Philadelphia, PA, pp. 102-111 (2007)

16. Beck, H.: Evolution of database designs for knowledge management in agriculture and natural resources. Journal of Information Technology in Agriculture 3(1) (2008)

17. Miller, G.A.: Wordnet, a lexical database for the english language, http://wordnet.princeton.edu/

18. W3C Committee: OWL Web Ontology Language Reference (February 2004), http://www.w3.org/TR/owl-ref/

19. Guo, Y., Pan, Z., Heflin, J.: LUBM: A Benchmark for OWL Knowledge Base Systems. Journal of Web Semantics 3(2), 158-182 (2005)

20. OWL-S Coalition: OWL-S: Semantic Markup for Web Services (2003), http://www.daml.org/services/owl-s/1.0/owl-s.html

21. Paolucci, M., Kawmura, T., Payne, T., Sycara, K.: Semantic matching of web services capabilities. In: First International Semantic Web Conference (2002)

22. Fensel, D., Lausen, H., de Bruijn, A.P.J., Stollberg, M., Domingue, D.R.J.: Enabling Semantic Web Services, 1st edn. Springer, Heidelberg (2007)

23. Semantic Web Services Committee: Semantic web services language (swsl) (2005), http://www.w3.org/Submission/SWSF-SWSL/

24. Grosof, B.N.: Silk: Higher level rules with defaults and semantic scalability. In: International Conference of Web Reasoning and Rule Systems, pp. 24-25 (2009)

25. Horrocks, I., Li, L., Turi, D., Bechhofer, S.: The instance store: Description logic reasoning with large numbers of individuals. In: IJCAR (2004)

26. Calvanese, D., Giacomo, G.D., Lembo, D., Lenzerini, M., Rosati, R.: Dl-lite: Tractable description logics for ontologies. In: AAAI (2005)

27. Dolby, J., Fokoue, A., Kalyanpur, A., Kershenbaum, A., Schonberg, E., Srinivas, K., Ma, L.: Scalable semantic retrieval through summarization and refinement. In: AAAI (2007) 